\title{
Homotopy Analysis Method for a Conservative Nonlinear Oscillator with Fractional Power
}

\author{
Huaxiong Chen, Yanyan Wang \\ School of Mathematics and Statistics, Zhoukou Normal University, Zhoukou, China \\ Email: chen410401301@163.com
}

How to cite this paper: Chen, H.X. and Wang, Y.Y. (2021) Homotopy Analysis Method for a Conservative Nonlinear Oscillator with Fractional Power. Journal of Applied Mathematics and Physics, 9, 31-40. https://doi.org/10.4236/jamp.2021.91004

Received: December 2, 2020

Accepted: January 3, 2021

Published: January 6, 2021

Copyright (อ 2021 by author(s) and Scientific Research Publishing Inc. This work is licensed under the Creative Commons Attribution International License (CC BY 4.0).

http://creativecommons.org/licenses/by/4.0/ (c) (i) Open Access

\begin{abstract}
In this paper, the homotopy analysis method is applied to deduce the periodic solutions of a conservative nonlinear oscillator for which the elastic force term is proportional to $u^{1 / 3}$. By introducing the auxiliary linear operator and the initial guess of solution, the homotopy analysis solving is set up. By choosing the suitable convergence-control parameter, the accurate high-order approximations of solution and frequency for the whole range of initial amplitudes can easily be obtained. Comparison of the results obtained using this method with those obtained by different methods reveals that the former is more accurate, effective and convenient for these types of nonlinear oscillators.
\end{abstract}

\section{Keywords}

Homotopy Analysis Method, Approximate Solutions, Fractional Power, Nonlinear Oscillation

\section{Introduction}

Classical perturbation methods including the Lindstedt-Poincaré method, the Krylov-Bogoliubov-Mitropolski method and the multiple scales method, as described by Nayfeh [1] [2], Kevorkian and Cole [3] and Verhulst [4], are limited to the weakly nonlinear systems. Thus, to extend the classical perturbation methods to the strongly nonlinear systems has been the desire of researchers for a long time.

During the past few decades, based on the classical ones, many improved or innovative methods applicable to the strongly nonlinear systems have been developed in open literature. Such as the modified Lindstedt-Poincaré method [5], the hyperbolic Lindstedt-Poincaré method [6], the incremental harmonic balance method [7], the perturbation-incremental method [8], the homotopy anal- 
ysis method [9], etc.

Among these strongly nonlinear methods, the HAM (the abbreviation for homotopy analysis method) proposed by Liao in 1992 [9] is a general analytical method. The advantage of the HAM as compared to other perturbation methods is that the HAM is independent of the small/large parameters. More importantly, by introducing the non-zero convergence-control parameter $h$ and the nonzero auxiliary function $H(t)$, this method provides a simple way to control and to ensure the convergence of approximation series. Another advantage of the HAM is that one can construct a continuous mapping of the initial guess approximation to the exact solution of the given problem through an auxiliary linear operator. Hence, the HAM attracts a great deal of interest of more and more researchers and has found applications in various nonlinear problems. On the basic ideas and the applications of HAM, one can refer to [9] [10] [11].

In this paper, Liao's HAM is applied to obtain the high-order analytical periodic solutions of a conservative nonlinear oscillator for which the elastic force term is proportional to $u^{1 / 3}$. This nonlinear oscillator has been recently studied by Beléndez [12] [13] using the HPM (the abbreviation for homotopy perturbation method), and by Lim [14] and Mickens [15] using the HBM (the abbreviation for harmonic balance method). By introducing the auxiliary linear operator and the initial guess of solution, the homotopy analysis solving for this nonlinear oscillator is set up, the high-order approximations of frequency and solution can easily be obtained. Excellent agreement of the approximate frequency with the exact one has been demonstrated and discussed, and we show that the secondorder approximation for frequency in this paper is better than the results by the preceding methods, the maximal relative error of the sixth-order approximate frequency by HAM is less than $0.0045 \%$. As can be seen, the results presented in this paper reveal that the HAM is very effective and convenient for conservative nonlinear oscillators with non-polynomial elastic terms.

\section{Solution Procedure}

Considering the following nonlinear oscillator which was introduced as a model "truly nonlinear oscillator" by Mickens [15] [16] [17]

$$
\ddot{u}+u^{1 / 3}=0,
$$

with initial conditions

$$
u(0)=A, \dot{u}(0)=0 .
$$

where the over-dot denotes differentiation with respect to time $t$ and $A$ is the amplitude of the oscillation. System (1) is a conservative nonlinear oscillator with a fractional power restoring force, it is not amenable to exact treatment and, therefore, the HAM can be applied to solve it. In order to apply the HAM effectively, we rewrite (1) in the following form

$$
(i i)^{3}+u=0 .
$$


By introducing the new variables

$$
u(t)=A y(t), \tau=\omega t,
$$

Equation (3) becomes

$$
\Omega A^{2} y^{\prime \prime 3}+y=0,
$$

with initial conditions

$$
y(0)=1, y^{\prime}(0)=0 .
$$

where $\Omega=\omega^{6}$, prime denotes the derivative with respect $\tau$, and $\omega$ represents the angular frequency.

According to [12], the periodic solution of (5) does exist, the solution $y(\tau)$ can be expressed by such a set of base functions

$$
\{\cos (2 m-1) \tau \mid m=1,2,3, \cdots\},
$$

that

$$
y(\tau)=\sum_{m=1}^{\infty} \alpha_{m} \cos (2 m-1) \tau .
$$

where $\alpha_{m}$ are coefficients that should be determined. Considering the initial conditions (6) and the rule of solution expression described by (8), a good initial guess of $y(\tau)$ can be determined as

$$
y_{0}(\tau)=\cos (\tau),
$$

According to Liao [9], the auxiliary linear operator can be given easily

$$
L(Y)=Y^{\prime \prime}+Y .
$$

Now, combining (5) with (10), we can construct the zeroth-order deformation equation

$$
(1-p) L\left[Y(\tau)-y_{0}(\tau)\right]=h H(\tau) p N[Y(\tau, p), \Lambda(p)],
$$

with the initial conditions

$$
Y(0, p)=1, Y^{\prime}(0, p)=0 .
$$

where the non-zero constant $h$ is called the convergence-control parameter, $p \in[0,1]$ is called the homotopy parameter, $H(\tau)$ is called the non-zero auxiliary function, and

$$
N[Y(\tau, p), \Lambda(p)]=A^{2} \Lambda(p)\left[Y^{\prime \prime}(\tau, p)\right]^{3}+Y(\tau, p),
$$

is the nonlinear operator.

When $p=0$, we have $Y(\tau)=y_{0}(\tau)$. When $p=1$, the system (11) becomes (5). Thus, by ranging $p$ from $p=0$ to $p=1$, it turns out that the initial guess of solution (9) deforms continuously to the exact solution of system (5) provided the following series

$$
Y(\tau, p)=y_{0}(\tau)+\sum_{i=1}^{\infty} p^{i} y_{i}(\tau)
$$




$$
\Lambda(p)=\Omega_{0}+\sum_{i=1}^{\infty} p^{i} \Omega_{i}
$$

converge when $p=1$. The series in (14), (15) are called the homotopy series, in which, $y_{0}(\tau)$ is defined in (9). By selecting the convergence-control parameter $h$ suitably, in general, the convergence of the series (14), (15) can be guaranteed. If the series (14), (15) are convergent at $p=1$, then the exact solution of system (5) turn out to be

$$
\begin{gathered}
Y(\tau, 1)=y_{0}(\tau)+\sum_{i=1}^{\infty} y_{i}(\tau), \\
\Lambda(1)=\Omega_{0}+\sum_{i=1}^{\infty} \Omega_{i} .
\end{gathered}
$$

Then, the solution of (1) can be expressed as $u(t)=A Y(\tau, 1)$ and $\omega=\Omega=[\Lambda(1)]^{1 / 6}$.

With the homotopy series (14), (15), according to Liao [9], we get the higher-order deformation equation

$$
L\left[y_{n}(\tau)-\chi_{n} y_{n-1}(\tau)\right]=h H(\tau) R(\tau),
$$

which satisfies the initial conditions

$$
y_{n}(0)=0, y_{n}^{\prime}(0)=0 \text {. }
$$

in which

$$
\chi_{n}=\left\{\begin{array}{l}
0, n \leq 1, \\
1, n>1,
\end{array}\right.
$$

and

$$
\begin{aligned}
R(\tau) & =\frac{1}{(n-1) !} \frac{\mathrm{d}^{n-1} N[Y(\tau, p), \Lambda(p)]}{\mathrm{d} p^{n-1}} \\
& =\sum_{i=0}^{n-1} \sum_{j=0}^{n-1-j} \sum_{k=0}^{n-1-j-k} A^{2} \Omega_{i} y_{j}^{\prime \prime}(\tau) y_{k}^{\prime \prime}(\tau) y_{n-i-j-k}^{\prime \prime}(\tau)+\sum_{i=0}^{n-1} y_{i}(\tau) .
\end{aligned}
$$

By balancing the like power of $p$ in (18) and choosing $H(\tau)=1$ for convenience, it turns out that, when $n=1$

$$
y_{1}^{\prime \prime}+y_{1}=(1+h) y_{0}+y_{0}^{\prime \prime}+A^{2} \Omega_{0} h y_{0}^{\prime \prime 3},
$$

when $n=2$

$$
y_{2}^{\prime \prime}+y_{2}=(1+h) y_{1}+y_{0}^{\prime \prime}+A^{2} \Omega_{1} h y_{0}^{\prime \prime 3}+3 A^{2} h \Omega_{0} y_{0}^{\prime \prime 2} y_{1}^{\prime \prime},
$$

when $n=3$

$$
\begin{aligned}
y_{3}^{\prime \prime}+y_{3}= & (1+h) y_{2}+y_{2}^{\prime \prime}+A^{2} \Omega_{2} h y_{0}^{\prime \prime 3}+3 A^{2} h \Omega_{1} y_{0}^{\prime \prime 2} y_{1}^{\prime \prime} \\
& +3 A^{2} h \Omega_{0} y_{0}^{\prime \prime}\left(y_{1}^{\prime \prime}\right)^{2}+3 A^{2} h \Omega_{0}\left(y_{0}^{\prime \prime}\right)^{2} y_{2}^{\prime \prime} .
\end{aligned}
$$

Substituting (9) into (22) gives

$$
y_{1}^{\prime \prime}+y_{1}=\frac{4 h-3 A^{2} h \Omega_{0}}{4} \cos (\tau)-\frac{A^{2} h \Omega_{0}}{4} \cos (3 \tau) .
$$

The elimination of the secular term in (25) requires 


$$
\frac{4 h-3 A^{2} h \Omega_{0}}{4}=0
$$

yields

$$
\Omega_{0}=\frac{4}{3 A^{2}} .
$$

According to (19) and (25), we can obtain

$$
y_{1}(\tau)=\frac{h \cos (3 \tau)-h \cos (\tau)}{24} .
$$

Substituting (9), (27) and (28) into (23) gives

$$
\begin{aligned}
y_{2}^{\prime \prime}+y_{2}= & \frac{-7 h^{2}-18 A^{2} h \Omega_{1}}{24} \cos (\tau)-\frac{8 h+16 h^{2}+6 A^{2} h \Omega_{1}}{24} \cos (3 \tau) \\
& -\frac{3 h^{2}}{8} \cos (5 \tau),
\end{aligned}
$$

No secular term in (29) requires

$$
\frac{-7 h^{2}-18 A^{2} h \Omega_{1}}{24}=0,
$$

yields

$$
\Omega_{1}=-\frac{7 h}{18 A^{2}}
$$

Solving (29) gives

$$
y_{2}=-\frac{12 h+25 h^{2}}{288} \cos (\tau)+\frac{24 h+41 h^{2}}{576} \cos (3 \tau)+\frac{h^{2}}{64} \cos (5 \tau) .
$$

Substituting the known terms into (24) gives

$$
\begin{aligned}
y_{3}^{\prime \prime}+y_{3}= & -\frac{84 h^{2}+187 h^{3}+216 A^{2} h \Omega_{2}}{288} \cos (\tau) \\
& -\frac{96 h+356 h^{2}+359 h^{3}+72 A^{2} h \Omega_{2}}{288} \cos (3 \tau) \\
& -\frac{216 h^{2}+405 h^{3}}{288} \cos (5 \tau)-\frac{17 h^{3}}{32} \cos (7 \tau),
\end{aligned}
$$

Elimination of the secular term of (33) yields

$$
\Omega_{2}=-\frac{84 h+187 h^{2}}{216 A^{2}} .
$$

With the perturbation procedure described above going on, the higher-order approximations for $\Omega_{n-1}$ and $y_{n}(\tau)(n>2)$ can be derived step by step. Hence, the series (14), (15) are derived.

By taking the transformations (4) into account, the approximations of frequency and solution of system (1) can be written in the following form

$$
\begin{gathered}
u(t)=A Y(\tau, 1)=A y_{0}(\omega t)+\sum_{i=1}^{\infty} A y_{i}(\omega t), \\
\omega=[\Lambda(1)]^{1 / 6}=\left[\Omega_{0}+\sum_{i=1}^{\infty} \Omega_{i}\right]^{1 / 6} .
\end{gathered}
$$




\section{Results and Discussion}

In this section, the HAM is applied to obtain the frequency and periodic solution of a conservative nonlinear oscillator for which the elastic force term is proportional to $u^{1 / 3}$ and its accuracy and efficiency are illustrated by comparing the approximate frequency obtained by HAM with the exact one $\omega_{e x}$ and other results in the literature.

The exact frequency for system (1) is given by the following expression [12]

$$
\omega_{e x}=\frac{2 \pi \Gamma(5 / 4)}{\sqrt{6} \Gamma(3 / 4) \Gamma(1 / 2) A^{1 / 3}}=\frac{1.070451}{A^{1 / 3}} .
$$

Figure 1 illustrates the effect of convergence-control parameter $h$ on the $A^{2} \Omega$ for different order approximation of HAM solutions. It is obvious that with the increases of the order of approximation, the $A^{2} \Omega$ becomes independent of $h$ and remains fixed.

From Figure 1, according to Liao [9], the derivative of $A^{2} \Omega$ with respect to $h$ is 0 . So, we can choose $h=-0.449198$. Under this condition, the second-order approximations of frequency and solution of system (1) are

$$
\begin{gathered}
u^{(2)}(t)=1.01992 A \cos \left(\omega_{2} t\right)-0.0230704 A \cos \left(3 \omega_{2} t\right) \\
+0.00315279 A \cos \left(5 \omega_{2} t\right), \\
\omega_{2}=\frac{1.07086}{A^{1 / 3}},
\end{gathered}
$$

therefore, it can be easily obtained that the relative error of frequency is $0.039 \%$, that is, the second-order approximations give the frequency with the highest accuracy. This indicates that the convergence-control parameter $h$ plays an important role in the HAM.

Figures 2-4 show the displacement $u(t)$ of system (1) obtained by (38) and (39) for amplitudes $A=0.001,1$ and 100. It can be seen from these figures that

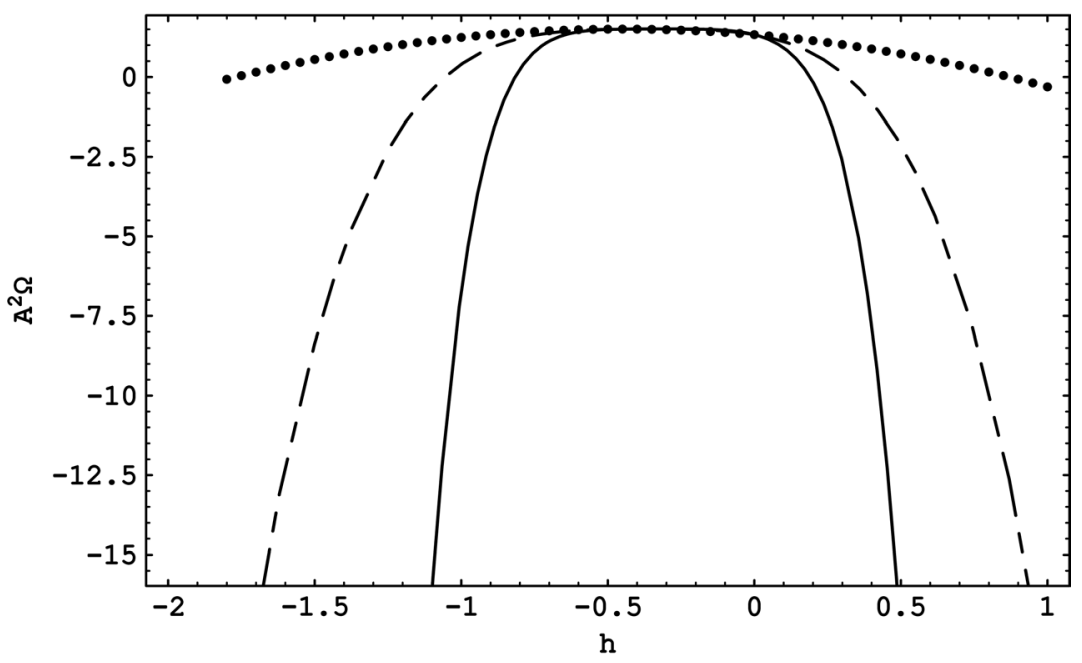

Figure 1. The effect of convergence-control parameter $h$ on $A^{2} \Omega ; \ldots 2^{\text {nd }}$-order approximation, --- $4^{\text {th }}$-order approximation, $-6^{\text {th }}$-order approximation. 


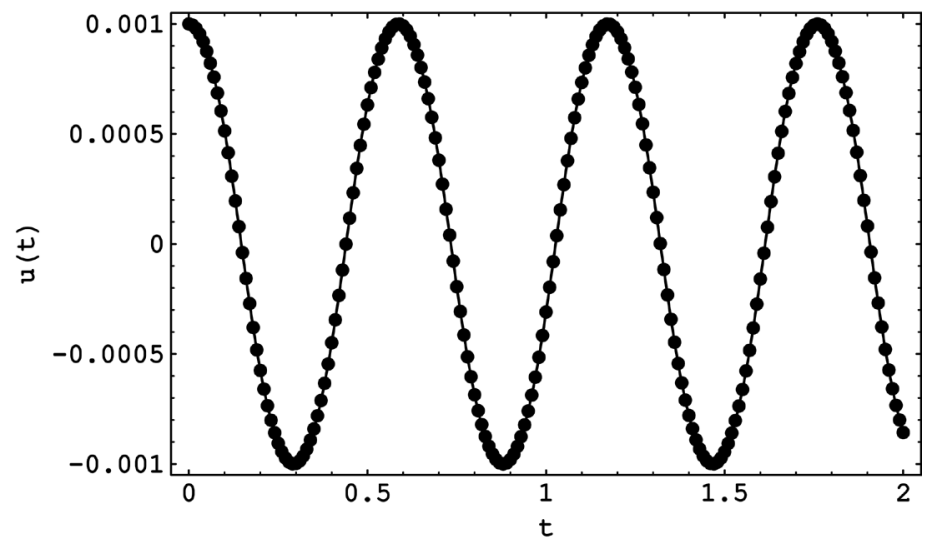

Figure 2. Displacement $u(t)$ versus time $t$ for $A=0.001$; $\cdots$ exact solution, $-2^{\text {nd }}$-order approximation of HAM.

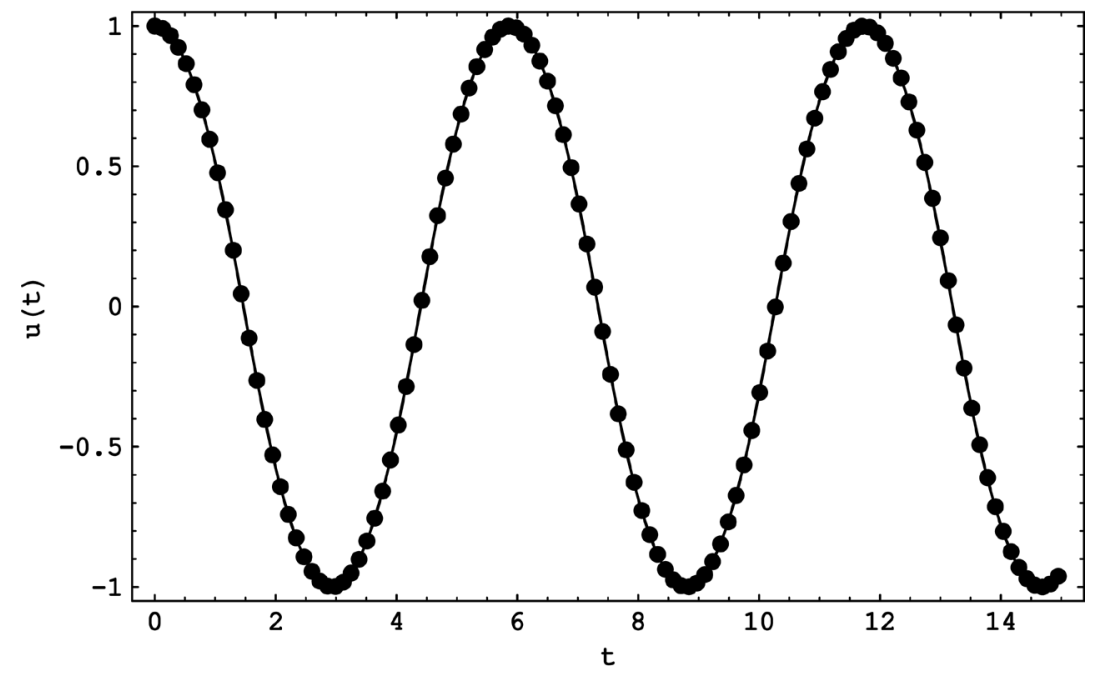

Figure 3. Displacement $u(t)$ versus time $t$ for $A=1$; $\bullet \bullet$ exact solution, $-2^{\text {nd }}$-order approximation of HAM.



Figure 4. Displacement $u(t)$ versus time $t$ for $A=100 ; \cdot \bullet$ exact solution, $-2^{\text {nd }}$-order approximation of HAM. 
Table 1. Comparison of the exact and approximate frequencies $\left(2^{\text {nd }}\right.$-order $)$ obtained using different methods.

\begin{tabular}{cccccc}
\hline & HAM (this paper) & HPM [12] & HPM [13] & HBM [14] & HBM [15] \\
\hline$A^{1 / 3} \omega_{2}(\%$ error) & $1.07086(0.039 \%)$ & $1.06861(0.17 \%)$ & $1.06991(0.050 \%)$ & $1.06928(0.11 \%)$ & $1.06341(0.66 \%)$ \\
\hline
\end{tabular}

the HAM provides excellent approximations to the exact periodic solution for the wide range of initial amplitudes for this case study.

In Table 1, we present the comparison between the approximate and exact frequency for the second-order approximation using different methods. It is clear that for the second-order approximation, the result obtained by HAM is better than those obtained previously by other authors.

In order to make the solutions more accurate, we get the higher-order approximations easily by the perturbation procedure described in section 2. Here, the sixth-order approximations of frequency and solution can be expressed in the following

$$
\begin{aligned}
& u^{(6)}(t)= 1.02054 A \cos \left(\omega_{6} t\right)-0.0242185 A \cos \left(3 \omega_{6} t\right) \\
&+0.00467294 A \cos \left(5 \omega_{6} t\right)-0.00159212 A \cos \left(7 \omega_{6} t\right) \\
&+0.000784884 A \cos \left(9 \omega_{6} t\right)-0.000320245 A \cos \left(11 \omega_{6} t\right) \\
&+ 0.000131784 A \cos \left(13 \omega_{6} t\right), \\
& \omega_{6}=\frac{1.070499}{A^{1 / 3}} .
\end{aligned}
$$

the relative error of frequency is $0.00448 \%$, that is, the sixth-order approximations are more accurate than the second-order ones.

\section{Conclusions}

1) The homotopy analysis method is applied to deduce the periodic solutions of a conservative nonlinear oscillator for which the elastic force term is proportional to $u^{1 / 3}$. The noteworthy feature of this method is its high accuracy for the whole range of values of oscillation amplitude. Moreover, the HAM solution can be quickly convergent by choosing the suitable convergence-control parameter and its calculation is very simple. Also, compared to other results by different methods, it can be shown that the HAM is very accurate, effective and convenient and has a great potential to be applied to other strongly nonlinear oscillators.

2) By using computer algebraic system: MATHEMATICA, the symbol deductions can be implemented easily.

\section{Acknowledgements}

This article was supported by the Key Research Projects in University of Henan Province (19A110038) and the Research Fund Projects of Zhoukou Normal University (ZKNUC2016012). 


\section{Conflicts of Interest}

The authors declare no conflicts of interest regarding the publication of this paper.

\section{References}

[1] Nayfeh, A.H. (1973) Perturbation Methods. Wiley, New York.

[2] Nayfeh, A.H. (1981) Introduction to Perturbation Methods. Wiley, New York.

[3] Kevorkian, J.K. and Cole, J.D. (1981) Perturbation Methods in Applied Mathematics. Springer-Verlag, New York. https://doi.org/10.1007/978-1-4757-4213-8

[4] Verhulst, F. (2005) Methods and Applications of Singular Perturbations: Boundary Layers and Multiple Timescales Dynamics. Springer-Verlag, New York. https://doi.org/10.1007/0-387-28313-7

[5] Cheung, Y.K., Chen, S.H. and Lau, S.L. (1991) A Modified Lindstedt-Poincaré Method for Certain Strongly Non-Linear Oscillators. Nonlinear Dynamics, 26, 367-378. https://doi.org/10.1016/0020-7462(91)90066-3

[6] Chen, Y.Y. and Yan, L.W. (2012) Heteroclinic Bifurcation Analysis of Duffing-Van Der Pol System by the Hyperbolic Lindstedt-Poincaré Method. Advanced Materials Research, 538-541, 2654-2657. https://doi.org/10.4028/www.scientific.net/AMR.538-541.2654

[7] Lau, S. and Cheung, Y. (1981) Amplitude Incremental Variational Principle for Nonlinear Vibration of Elastic Systems. Journal of Applied Mechanics, 48, 959. https://doi.org/10.1115/1.3157762

[8] Chung, K.W., Chan, C.L., Xu, Z. and Xu, J. (2005) A Perturbation-Incremental Method for Strongly Non-Linear Non-Autonomous Oscillators. International Journal of Non-Linear Mechanics, 40, 845-859. https://doi.org/10.1016/j.ijnonlinmec.2004.10.002

[9] Liao, S.J. and Sherif, S. (2004) Beyond Perturbation: Introduction to the Homotopy Analysis Method. Applied Mechanics Reviews, 57, 25. https://doi.org/10.1115/1.1818689

[10] Liao, S.J. and Tan, Y.A. (2007) A General Approach to Obtain Series Solutions of Nonlinear Differential Equations. Studies in Applied Mathematics, 119, 297-354. https://doi.org/10.1111/j.1467-9590.2007.00387.x

[11] Liao, S.J. (2009) Notes on the Homotopy Analysis Method: Some Definitions and Theorems. Communications in Nonlinear Science \& Numerical Simulation, 14, 983-997. https://doi.org/10.1016/j.cnsns.2008.04.013

[12] Beléndez, A., Pascual, C., Gallego, S., OrtuO, M. and Neipp, C. (2007) Application of a Modified He's Homotopy Perturbation Method to Obtain Higher-Order Approximations of an $x^{1 / 3}$ Force Nonlinear Oscillator. Physics Letters A, 371, 421-426. https://doi.org/10.1016/j.physleta.2007.06.042

[13] Beléndez, A. (2009) Homotopy Perturbation Method for a Conservative $x^{1 / 3}$ Force Nonlinear Oscillator. Computers and Mathematics with Applications, 58, 22672273. https://doi.org/10.1016/j.camwa.2009.03.011

[14] Lim, C.W. and Wu, B.S. (2005) Accurate Higher-Order Approximations to Frequencies of Nonlinear Oscillators with Fractional Powers. Journal of Sound \& Vibration, 281, 1157-1162. https://doi.org/10.1016/j.jsv.2004.04.030

[15] Mickens, R.E. (2001) Oscillations in an $x^{4 / 3}$ Potential. Journal of Sound \& Vibration, 246, 375-378. https://doi.org/10.1006/jsvi.2000.3583 
[16] Mickens, R.E. (2002) Analysis of Non-Linear Oscillators Having Nonpolynomial Elastic Terms. Journal of Sound \& Vibration, 255, 789-792.

https://doi.org/10.1006/jsvi.2001.4172

[17] Cooper, K. and Mickens, R.E. (2002) Generalized Harmonic Balance/Numerical Method for Determining Analytical Approximations to the Periodic Solutions of the $x^{4 / 3}$ Potential. Journal of Sound \& Vibration, 250, 951-954.

https://doi.org/10.1006/jsvi.2001.3782 